\title{
Fiber-optic temperature sensors based on differential spectral transmittance/reflectivity and multiplexed sensing systems
}

\author{
Anbo Wang, George Z. Wang, Kent A. Murphy, and Richard O. Claus
}

\begin{abstract}
A concept for optical temperature sensing based on the differential spectral reflectivity/transmittance from a multilayer diel ectric edge filter is described and demonstrated. Two wavel engths, $\lambda_{1}$ and $\lambda_{2}$, from the spectrum of a broadband light source are selected so that they are located on the sloped and flat regions of the reflection or transmission spectrum of the filter, respectively. As temperature variations shift the reflection or transmission spectrum of the filter, they change the output power of the light at $\lambda_{1}$, but the output power of the light at $\lambda_{2}$ is insensitive to the shift and therefore to the temperature variation. The temperature information can be extracted from the ratio of the light powers at $\lambda_{1}$ to the light at $\lambda_{2}$. This ratio is immune to changes in the output power of the light source, fiber losses induced by microbending, and hence modal-power distribution fluctuations. The best resolution of $0.2^{\circ} \mathrm{C}$ has been obtained over a range of $30-120^{\circ} \mathrm{C}$. Based on such a basic temperature-sensing concept, a wavel ength-division-multiplexed, temperature-sensing system is constructed by cascading three sensingedge filters that have different cutoff wavelengths along a multimode fiber. The signals from the three sensors are resolved by detecting the correspondent outputs at different wavel engths.
\end{abstract}

\section{Introduction}

Many optical-fiber sensors have been demonstrated in laboratories during the past 15 years to measure a wide range of physical parameters. ${ }^{1}$ Generally fiber sensors may be classified into two classes: interferometric devices that measure differential phasechanges in multipath fiber geometries and intensity-based devices that measure changes in received optical power. Extremely high resolution, $10^{-6}$ rad, has been reported from the use of fiber interferometers; however, they require relatively complicated signalprocessing techniques for the effective phase recovery to be accomplished. This greatly increases their cost and makes them unacceptable for many industrial system applications. In addition to these drawbacks, the absolute measurement with high speed has not yet become a reality for such sensors. I ntensitybased fiber sensors conversely require simple signal

The authors are with the Fiber and Electro-Optics Research Center, Bradley Department of Electrical Engineering, Virginia Polytechnic Institute and State University, Blacksburg, Virginia 24061-0111.

Received 1J une 1993; revised manuscript received 14 September 1994.

0003-6935/95/132295-06\$06.00/0.

(c) 1995 Optical Society of America. processing while possessing all the normal advantages of fiber sensors over conventional electricsensing techniques, namely, immunity to electromagnetic interference, small size, geometric flexibility, multiplexability, and the possibility of use in harsh environments. However, the output readings of intensity-based fiber sensors are usually sensitive to changes in source power, fiber attenuation, and electronics. A recalibration process is required after the light source is replaced or the fiber is respliced for most of the intensity-based sensors. To overcome these problems, different self-referencing techniques have been proposed. In 1985 Giles et al. ${ }^{3}$ proposed and demonstrated an optical bridge compensation for intensity-based fiber sensors. A dual-wavelength operation scheme was proposed by Kersey et al. in 1989. ${ }^{4}$ Wlodarczyk et al. ${ }^{5}$ in 1991 used a dualwavelength scheme to compensate successfully for the temperature effect in their fiber-optic pressure sensor for combustion monitoring and control. In 1992 Berkcan $^{6}$ demonstrated optical-temperaturecompensation schemes of spectral modulation sensors for aircraft engine control. Recently Wang et al. ${ }^{7}$ proposed and demonstrated a compensation technique for polarimetric fiber sensors, and this method led to an industrial long-term, in-line operation for the measurement of pressure, oil storage, and liquid level. Because many mechanisms have been ex- 
ploited for sensing a wide variety of physical parameters, compensation technique development becomes the important factor for many practical operations of intensity-based fiber sensors.

Temperature measurement in particular is essential in a widerange of industrial and material fabrication processes. A number of different fiber temperature sensors have been demonstrated. For example, in 1983Alves et al. ${ }^{8}$ demonstrated a fiber temperature sensor based on the fluorescence decay-time variation of a phosphor layer. In 1983 Dils $^{9}$ proposed a fiberbased blackbody high-temperature sensor. Christensen and I ves ${ }^{10}$ devel oped a fiber temperature sensor using the temperature dependence of a GaAs absorption bandgap in 1987. Other basic concepts have also been proposed and demonstrated by many researchers in the past several years. ${ }^{11-14}$ In this paper we describe a fiber temperature sensor based on the temperature dependence of differential spectral transmission or reflection of dielectric multilayer filters and present the corresponding experimental results. A multiplexed sensing system containing three such filter-based temperature sensors is also described.

\section{Temperature Sensors}

The spectral transmission and reflection characteristics of a multilayer dielectric filter are determined by the refractive indices and thickness of the multiple layers, the number of layers, the angle of the incident light beam, and the refractive index of the media surrounding the filter. Because both the indices and thicknesses of the individual layer materials depend on temperature, the transmission or reflection spectrum is also a function of temperature. ${ }^{15,16}$ Thus, when a wavel ength $\lambda_{1}$ of incident light on the filter is located near the edge of the transmission or reflection spectrum passband of the filter, the intensity of the transmission or reflection will be a function of temperature, which is the basis of the operation of the sensors described in this paper. However, as addressed in Section 1, a major problem associated with intensity-based fiber sensors is the signal drifts caused by optical-power variations. To overcome this obstacle, a reference signal is selected in a way that its wavelength $\lambda_{2}$ is located in the flat region of the transmission or reflection spectral profile of the filter. This reference signal will be much less sensitive to temperature variations than the sensing signal, but the reference signal carries the same information about optical power changes caused by fiber attenuation and source-power variations if the two wavelengths are close enough to each other. Thus the ratio of the power of light at $\lambda_{1}$ to the power of light at $\lambda_{2}$, which is also a function of temperature, is immune to the changes in fiber loss, modal-power distribution (MPD) in the fiber, and source power.

\section{A. Transmission-Based Fiber Temperature Sensor}

A schematic of the sensor is shown in Fig. 1. Broadband light is launched into one end of a lead-in

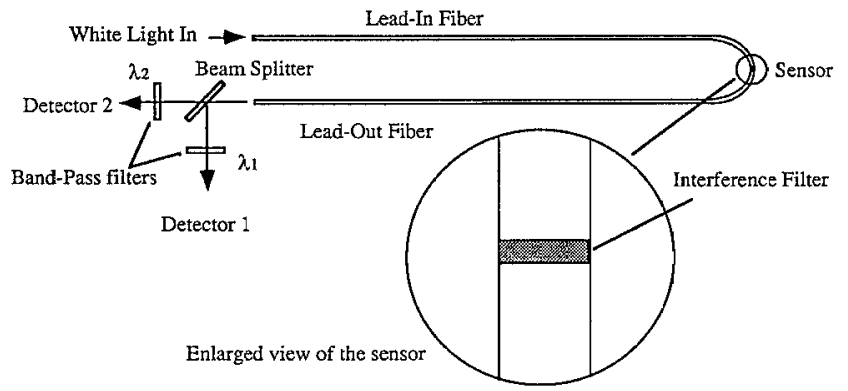

Fig. 1. Schematic of the transmission-based temperature sensor.

multimode fiber. The light from that fiber is transmitted through a dielectric multilayer edge filter. A multimodelead-out fiber receives the light transmitted through the filter. At the output end of the lead-out fiber, the optical signal is split into two beams that are filtered by two bandpass filters with central wavelengths $\lambda_{1}$ and $\lambda_{2}$. These two wavelengths are located in the sloped and flat regions of the transmission spectrum of the filter, respectively, as shown in Fig. 2. The sensor output signal is obtained from the power ratio of the two filtered signals.

We have constructed and tested the temperature sensor in our laboratory. A white-light source was used and two 100-140- $\mu \mathrm{m}$, graded-index glass fibers were employed as the lead-in and lead-out fibers. A short-wave-pass interference filter coated on a 0.9-mm-thick glass slide acted as the temperaturesensing el ement. The transmission spectrum of the filter is shown in Fig. 3. Temperature tests of the filter response indicated that its transmission spectrum shifted toward shorter wavelengths with an increase in temperature. To reduce the fiber-tofiber coupling loss at the location of the filter, we polished the filter down to a thickness of $0.4 \mathrm{~mm}$. The polished endfaces of the two multimode fibers were al igned and attached to the filter with an optical epoxy. Spectral bandpass filters centered at 633 and $650 \mathrm{~nm}$ with a spectral width of $\sim 10 \mathrm{~nm}$ were used to select the temperature-sensitive and insensitive output signals as shown in Fig. 3. Figure 4 shows the corresponding calibration curve of the sensor. Experimental tests indicated a resolution of $0.2{ }^{\circ} \mathrm{C}$ over

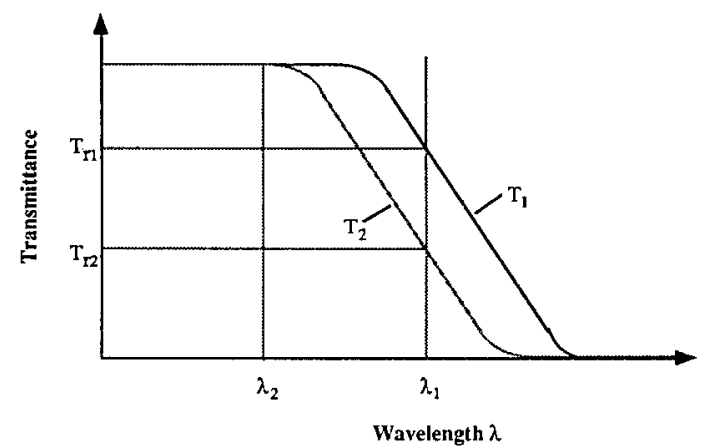

Fig. 2. Operation principle based on differential transmittance at two different temperatures, $T_{1}$ and $T_{2}$. 


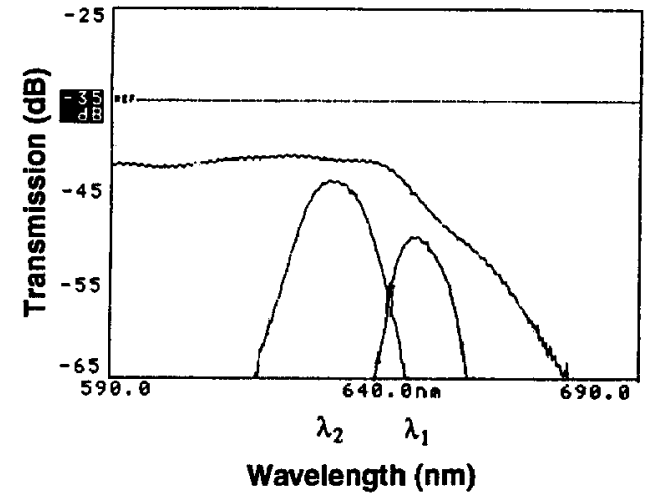

Fig. 3. Measured transmission spectrum of the short-wave-pass filter and bandpass filters.

a $30-120^{\circ} \mathrm{C}$ temperature range. Although the upper temperature limit of the filter was $\sim 300^{\circ} \mathrm{C}$, the epoxy bonding the fibers to the filter placed a limitation on the upper test temperature.

To protect the fiber-filter interfaces from damage and provide mechanical support to the sensor, we applied another epoxy (BIPAX TRA-BOND Type BAF113SC manufactured by TRA-CON) tothe fiber-filter junction. This packaging also prevented the edge filter from being exposed to the environment. $\mathrm{Be}$ cause we gradually heated the sensor in an oven (each experiment took $\sim 2 \mathrm{~h}$ to cover the temperature range from 30 to $120^{\circ} \mathrm{C}$ ), it was unlikely that the humidity in the atmosphere affected the sensor performance.

For most intensity-based fiber sensors the most serious problem typically is the dependence of the sensor output on the MPD in the fiber. To investigate the possible influence of such effects on the performance of this sensor, we modified the MPD in the lead-in fiber by bending the fiber, thus removing higher-order modes and causing a net decrease in the total transmitted optical power. The corresponding small changes in the output sensor signal amplitude are shown in Fig. 5. Similarly, Fig. 6 shows the variation in the sensor output caused by changes in

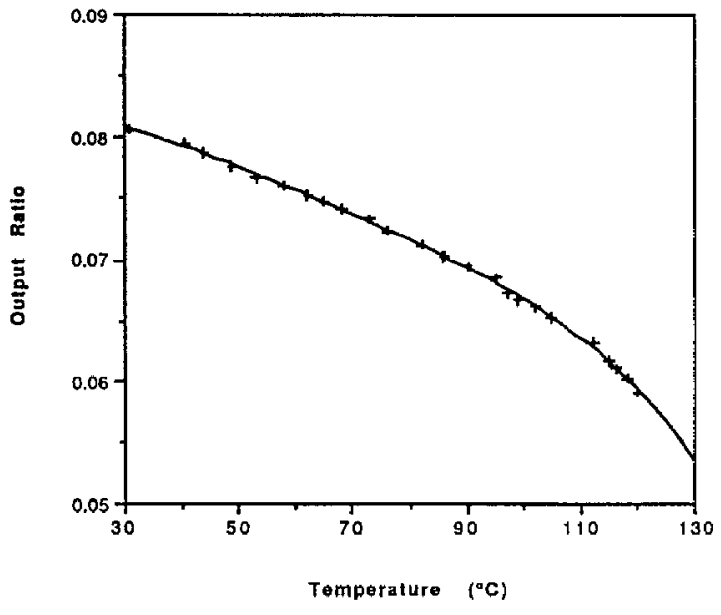

Fig. 4. Sensor output as a function of temperature.

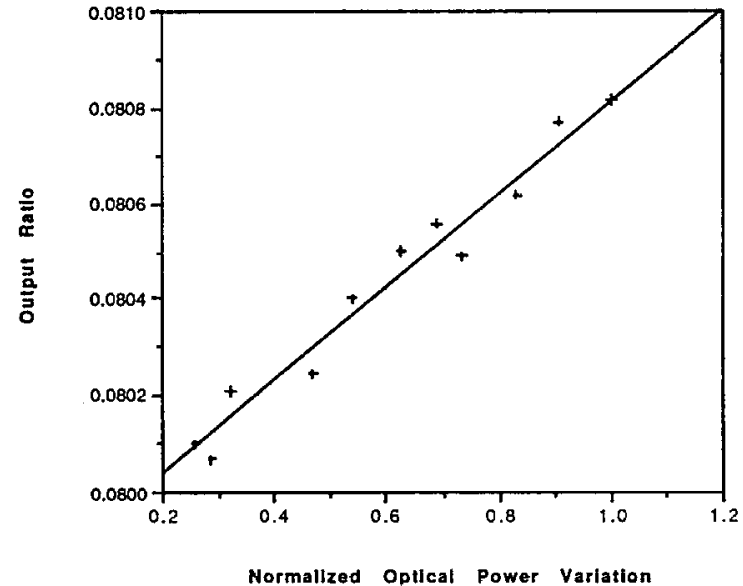

Fig. 5. Dependence of the sensor output on the fiber-bending loss.

the output power of the light source. The small changes in the sensor output indicate that this temperature sensor is effectively immune to the changes in source power and fiber-bending loss. Furthermore excellent repeatability was obtained for several temperature test cycles over the measurement range. No hysteresis has been observed within our experimental accuracy.

\section{B. Reflection-Based Fiber Temperature Sensor}

In a way similar to the transmission-based fiber temperature sensor described above, a reflectionbased fiber temperature sensor may also be constructed. A $2 \times 2$ directional fiber coupler is used to separate the optical signals reflected from the sensing filter. The schematic of the fiber temperature sensor based on the differential spectral reflectivity is shown in Fig. 7. Two wavelengths are selected so that they are located in the sloped and flat regions of the reflection spectrum of the filter. At the detector end the light at these two wavelengths is filtered by two bandpass filters. The sensor output is given by the power ratio of the light at the sensing wavelength to the light at the referencing wavelength to remove



Fig. 6. Dependence of the sensor output on the source-power variation.

1 May 1995 / Vol. 34, No. 13 / APPLIED OPTICS 




Fig. 7. Diagram of the reflection-based temperature sensor.

the influence from variations in fiber loss and lightsource power.

In experiments the same light source and the same type of fiber as in the previous experiments were used in the construction of the reflection-based temperature sensors. A multimode $2 \times 2$ coupler with a $50 / 50$ splitting ratio was used. Two dichroic shortwave-pass interference filters were tested for sensing temperature. The reflection spectra of these filters are shown in Fig. 8. According to the reflection spectra, two pairs of bandpass filters with central wavelengths of $671,647.1 \mathrm{~nm}$ and $620,590 \mathrm{~nm}$ were selected. Figure 9 shows the outputs of these two sensors as functions of temperatures. A resolution of $0.5^{\circ} \mathrm{C}$ has been obtained over a range of $50-100^{\circ} \mathrm{C}$.

\section{Multiplexed Fiber-Temperature-Sensing System}

Many multiplexing techniques for fiber-optic sensors have been devel oped ${ }^{17}$ in the past 10 years. They are divided mainly into four classes: timedivision multiplexing, frequency division multiplexing, wavelength division multiplexing, and coherence multiplexing. The sensors described in Subsections 2.A and 2.B are based on the differential spectral transmittance/reflectivity of short-wave-pass filters that have different cutoff wavelengths. One possible scheme to multiplex a number of such sensors is shown in Fig. 10. With this approach a number of sensors are cascaded along a single fiber. The signals from sensors at different wavelengths are selected from the reflections or both the reflections and transmission with a wavelength division demultiplexer. The transmission can be used only to obtain temperature information for the filter at the end farthest from the source.

In the corresponding experiment, three sensors described in Subsections 2.A and 2.B were multiplexed together by use of the approach shown in Fig.

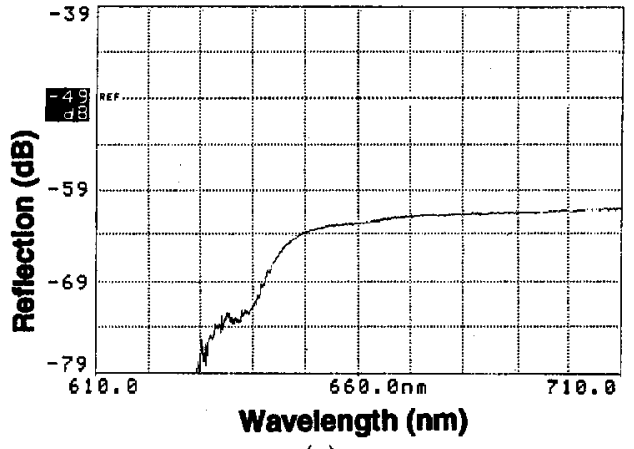

(a)

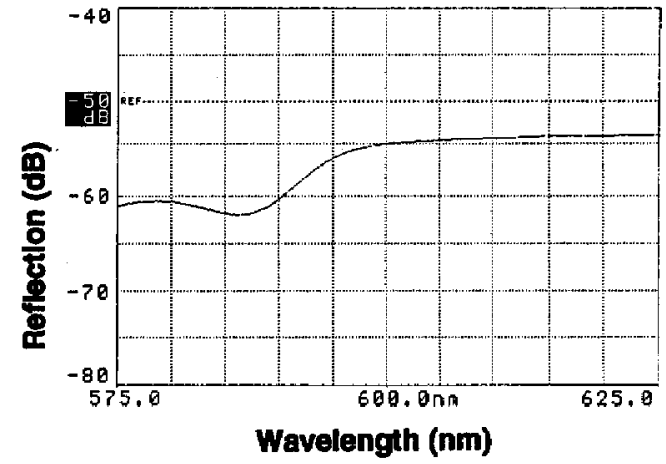

(b)

Fig. 8. Measured reflection spectra of the two short-wave-pass filters.

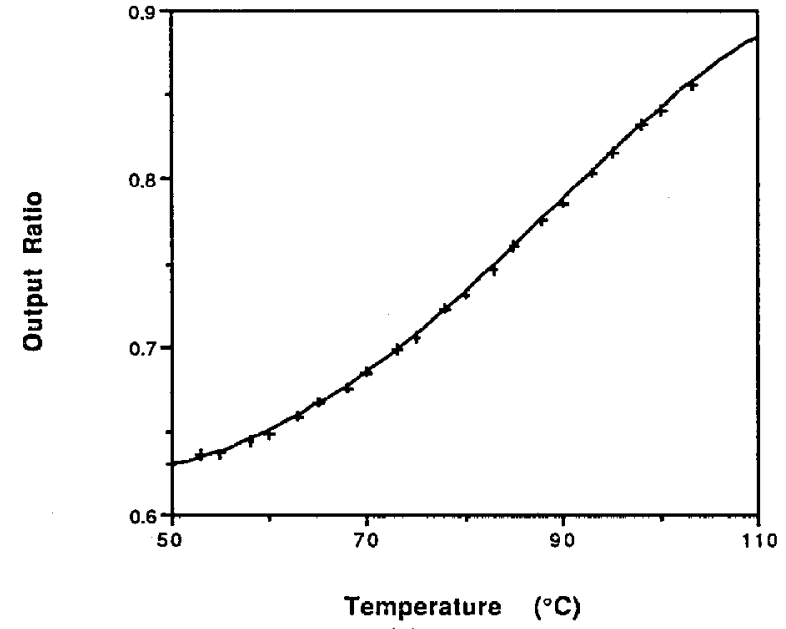

(a)

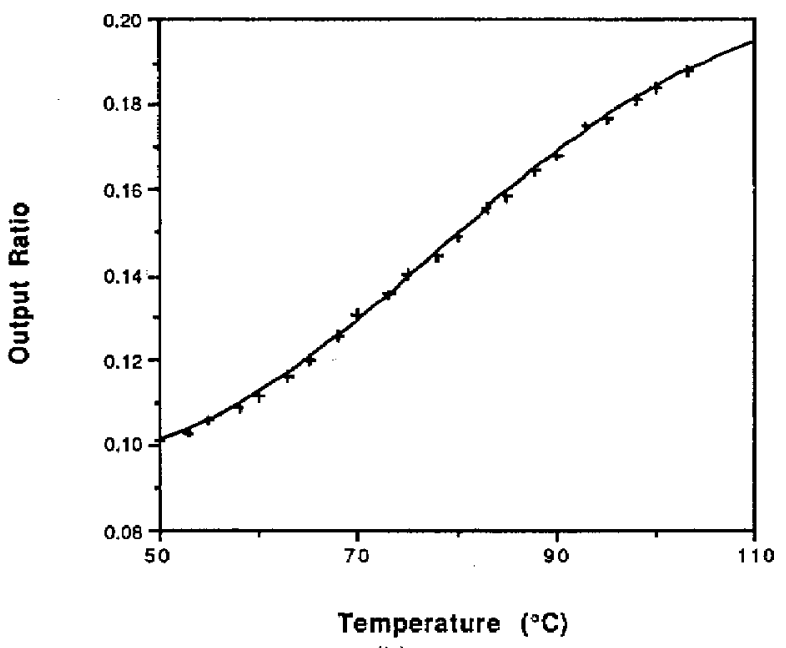

(b)

Fig. 9. Calibration curves of the reflection-based sensors: (a) sensor 1 with sensing and reference bandpass filters at 671 and $647.1 \mathrm{~nm}$, respectively; (b) sensor 2 with sensing and reference bandpass filters at 620 and $590 \mathrm{~nm}$, respectively. 
10 to examine the multiplexability of the sensors. Instead of with a wavelength division demultiplexer at the detector end, signals from a sensor were selected with a combination of a cubic beam splitter and two bandpass filters whose wavelengths were selected to be the same as the values in Sections 2.A and 2.B. To increase the signal-to-noise ratio (SNR) of the far-end sensor signal, transmission was monitored for this sensor. These three cascaded sensors were calibrated separately by gradually changing the temperature of each sensing filter while keeping the temperatures of the other two sensing filters constant. Note that the cutoff wavelengths of the sensing-edge filters multiplexed must obey the relation $\lambda_{1}>\lambda_{2}>$ $\lambda_{3}$. Figure 11 (the curves with the + symbols) shows the calibration curves.

Because all the multiplexed sensors were cascaded along one single fiber and the signals from different sensors were separated by monitoring the optical power at different wavelengths, cross talk may exist between the sensors. The effect was investigated by monitoring the signals of one sensor whose temperature was kept constant, while another sensor was heated. Figure 11 (the curves with the $\boldsymbol{\Delta}$ symbols) shows the cross-talk test results. As expected, the response is nearly flat, indicating that little cross talk took place.

\section{Discussion}

The optical-fiber, temperature-sensor output is given by the power ratio of two optical signals at two different wavelengths located in the flat and sloped regions of the transmission or reflection spectra of the sensing filter to compensate for variations in lightsource power and fiber-bending loss. The selection of the two operating wavelengths is important to sensor performance for several reasons, specifically to the long-term stability of the sensor. Fiber attenuation is related to MPD in the fiber. Because of the inherent properties of circular optical waveguides, different source wavelengths excite different MPD's in a fiber, although those different wavelengths are generated by the same source. Therefore, to obtain good compensation for fiber-loss variations, one should select the two wavelengths to be as close as possible. The spectral intensity distribution of a white-light source, for example, depends on the temperature of the lamp or its driving current and also may be dependent on the aging conditions of the lamp. The

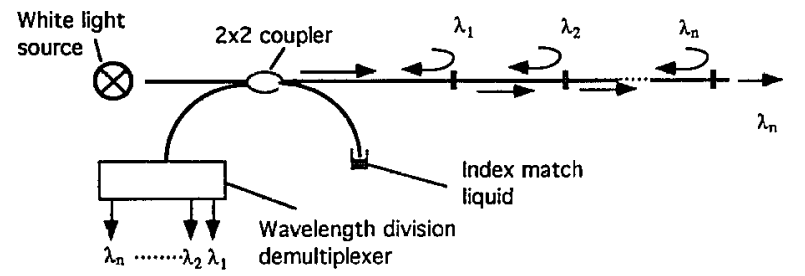

Fig. 10. Possible multiplexing scheme for the multilayer dielectric filter-based temperaturesensors. Wavelength-division-multiplexer-based cascading scheme.

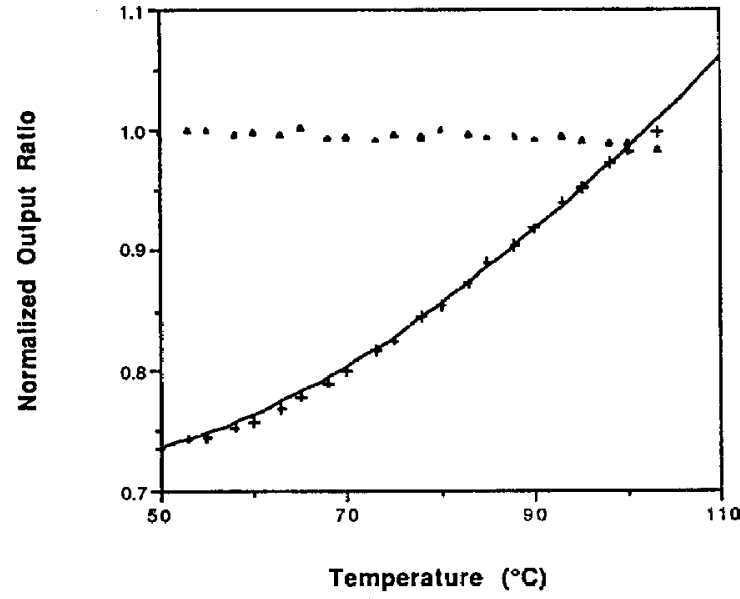

(a)

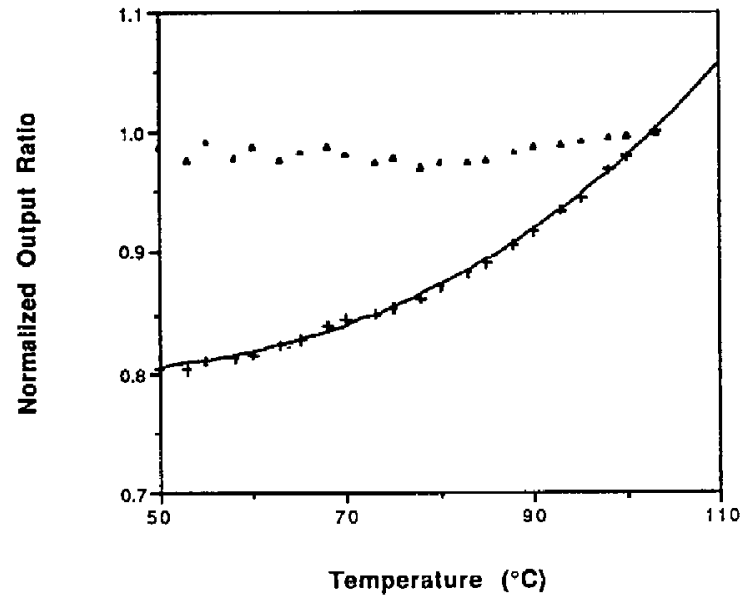

(b)



(c)

Fig. 11. Experimental results of the cross talk (compared with the sensor outputs) obtained by (a) heating sensor 2 and monitoring sensor 1 , (b) heating sensor 1 and monitoring sensor 2, and (c) heating sensor 1 and monitoring sensor 3 .

two wavelengths should be chosen so that both wavelengths are located on a flat region of the spectralintensity-distribution profile of the lamp. In this way, percentage changes in the intensity variations of 
the two signals at different wavelengths stay nearly the same during power variations of the light source.

We cascaded three edge filters al ong one singl e fiber to realize the multiplexed sensing system shown in Fig. 10. Studying the result of the cross-talk effect presented in Figure 11, we observed that the sensor output caused by the unwanted filters was nearly flat (unchanged). The cross talk was relatively small, and hence the capability of effectively multiplexing many filters to achieve a multiplexed sensing system is demonstrated. At the same time we also realize that the excess losses of the sensing filters between the light source and the monitored sensor are seen to be important to the SNR of the prototype sensor. We make these interference filters by depositing layers of dielectric materials onto glass substrates. Although the thickness of the filters was polished down to $150 \mu \mathrm{m}$ from an original value of $900 \mu \mathrm{m}$, this thickness still could cause significant insertion loss, especially when we consider that light signals pass through sensing el ements two and three times for the second and third edge filters, respectively. It is believed that the low SNR's of the two sensors were caused mainly by these thick glass substrates. We may remedy this problem by directly depositing the sensing filters onto fiber endfaces. Furthermore the direct deposition of the filter on the fiber endface may make it possible to build a temperature sensor whose diameter is below a few hundred micrometers, one that also has a fast time response. The practical spatial separation between two sensors in the multiplexed sensing system may be made as small as $1 \mathrm{~cm}$.

\section{Conclusions}

Dielectric-multilayer-filter-based, optical-fiber temperature sensors based on differential spectral transmittance/reflectivity have been demonstrated experimentally. A resolution of $0.2^{\circ} \mathrm{C}$ has been obtained over a measurement range of $30-120^{\circ} \mathrm{C}$. The sensor has been demonstrated to have low immunity to changes in light-source power and fiber-bending loss. A wavel ength-division-multiplexed sensing system has been constructed by cascading three such filters with different cutoff wavelengths al ong a single multimode fiber. A resolution of $0.5^{\circ} \mathrm{C}$ has been obtained over a temperature range of $50-100^{\circ} \mathrm{C}$. Cross talk between sensors has been investigated. The SNR of the multiplexed sensing system and hence resolution may be improved dramatically by directly depositing sensing filters onto fiber endfaces. The cross talk in the multiplexed sensing system may be reduced by using filters specifically designed for the multiplexed applications.

The authors thank Virginia Center for Innovative Technology and Fiber and Sensor Technologies, Inc., in Blacksburg, Va., for supporting this research.

\section{References}

1. E. Udd, Fiber Optic Sensors-an Introduction for Engineers and Scientists (Wiley, New York, 1991), Chap. 13, p. 409.

2. T. G. Giallorenzi, J . A. Bucardo, A. Dandridge, G. H. Siegel, J r., J. H. Cole, S. C. Rashleigh, and R. G. Priest, "Optical fiber sensor technology," IEEE J . Quantum Electron. QE-18, 626665 (1982).

3. I. P. Giles, S. McNeil, and B. Culshaw, "A stable remote intensity based fiber sensor," J . Phys. E 18, 1124-1126 (1985).

4. A. D. Kersey, M. A. Davis, and M. J . Marrone, "Differential polarimetric fiber-optic sensor configuration with dual wavelength operation,"Appl. Opt. 28, 204-206 (1989).

5. M. T. Wlodarczyk, D. Vokovich, V. Astrakhan, M. Kluzner, and O. Ulrich, "F iber optic pressure sensor for combustion monitoring and control," in Chemical, Biochemical, and Environmental Fiber Sensors III, M. T. Wlodarczyk, ed., Proc. Soc. PhotoOpt. Instrum. Eng. 1587, 4-14(1991).

6. E. Berkcan, "Optical temperature compensation schemes of spectral modulation sensors for aircraft engine control," Spe cialty Fiber Optic Systems for Mobile Platforms and Plastic Optical Fibers, L. Fegueroa, M. Katazawa, N. E. Lewis, R. Steele, and D. Varshneya, eds., Proc. Soc. Photo-Opt. Instrum. Eng. 1799, 149-158(1992).

7. A. Wang, S. He, X. Fang, X. J in, and J . Lin, "Optical fiber pressure sensor based on photoelasticity and its application," IEEE J . Lightwave Technol. 10, 1466-1471 (1992).

8. R. V. Alves, J. Christol, M. Sun, and K. A. Wickersheim, "Fluoroptic thermometry: temperature sensing using optical fibers," in Advances in Instrumentation, Glenn F. Harvey, ed. (Instrument Society of America, Houston, Tex., 1983), Vol. 38, pt. 2, pp. 925-932.

9. R. R. Dils, "High temperature optical fiber thermometer," J . Appl. Phys. 54, 1198-1201 (1983).

10. D. A. Christensen and J. T. Ives, "Fiber optic temperature probe using a semiconductor sensor," in Optical Fiber Sensors, A. N. Chester, S. Martellucci, A. M. verga Scheggi, eds., Proceedings of NATO Advanced Studies Institute Series, (Martinus Nijhoff, Dordrecht, The Netherlands, 1987), p. 361.

11. L. Schultheis, H. Amstrutz, and M. Kaufmann, "Fiber optic temperature sensing with ultrathin silicon étalons," Opt. Lett. 13, 782-784 (1988).

12. M. Gottlieb, G. B. Brandt, and J . Butler, "Measurement of temperature with optical fibers," ISA Trans. 19(4), 55-63 (1980).

13. A. Wang, G. Z. Wang, A. M. Vengsarkar, and R. O. Claus, "Elliptical-core two-mode fiber sensor for measurement of strain and temperature," in Fiber Optic and Laser Sensors IX, R. P. DePaula and E. Udd, eds., Proc. Soc. Photo-Opt. Instrum. Eng. 1584, 294-303 (1991).

14. A. H. Hartog, "A distributed temperature sensor based on liquid-core optical fibers," IEEE J . Lightwave Technol. LT-1, 498-509 (1983).

15. I. H. Blifford, J r., "F actors affecting the performance of commercial interference filters," Appl. Opt. 5, 105-111 (1966).

16. A. M. Title, T. P. Pope, and J. P. Andelin, J r., "Drift in interference filters. Part 1,"Appl. Opt. 13, 2675-2679(1974).

17. J. P. Dakin, The Distributed Fiber Optic Sensing Handbook (IFS Publication, Bedford, UK, 1990). 